\title{
Laparoscopic Conversion of One Anastomosis Gastric Bypass to a Standard Roux-en-Y Gastric Bypass
}

\author{
Imed Ben Amor ${ }^{1}$ - Niccolo Petrucciani ${ }^{1} \cdot$ Radwan Kassir $^{2}$ • Abdullah Al Munifi ${ }^{1}$. \\ Thierry Piche $^{1} \cdot$ Tarek Debs $^{1} \cdot$ Jean Gugenheim ${ }^{1}$
}

Published online: 16 March 2017

(C) Springer Science+Business Media New York 2017

\begin{abstract}
Introduction One anastomosis gastric bypass (OAGB) demonstrated results similar to traditional Roux-en-Y procedures [1-3], in terms of weight loss and resolution of obesity-related comorbidities. The main controversy regarding OAGB is the concern for an association between biliary alkaline gastritis and esophageal or gastric cancer raised by some studies [4].

Methods We present the case of a 51-year-old woman with a BMI of $41 \mathrm{~kg} / \mathrm{m} 2$ who underwent a laparoscopic OAGB in 2014. One year later, she consulted for recurrent heartburns. An upper GI endoscopy showed pouchitis and bile reflux in the esophagus. Medical treatment of gastroesophageal reflux disease was ineffective. We decided to convert the OAGB to a Roux-en-Y gastric bypass (RYGB).

Results In this video, we show how to revise an OAGB to treat chronic bile reflux, by converting the procedure to a standard RYGB. The intervention starts by restoring the normal anatomy of the small bowel, with the resection of the gastrojejunal anastomosis, which was located at 250-
\end{abstract}

Electronic supplementary material The online version of this article (doi:10.1007/s11695-017-2646-1) contains supplementary material, which is available to authorized users.

Niccolo Petrucciani nicpetrucciani@hotmail.it

1 Department of Digestive Surgery, Archet II Hospital, University of Nice Sophia Antipolis, 151 Route de Saint-Antoine, 06200 Nice, France

2 Department of General Surgery, CHU Hospital, Jean Monnet University, Avenue Albert Raimond, 42270 Saint Etienne, France $\mathrm{cm}$ du Treitz's ligament. Then, the gastric pouch is created. A standard Roux-en-Y gastric bypass is performed.

Conclusions The resection of the gastrojejunal anastomosis allows fashioning the Roux-en-Y limb with the classical measures. This technique allows a conversion to a standard RYGB and is effective in treating the biliary reflux.

Keywords Surgical technique $\cdot$ Roux-en-Y gastric bypass $\cdot$ Mini gastric bypass $\cdot$ Omega loop gastric bypass $\cdot$ Conversion · Gastroesophageal reflux

\section{Compliance with Ethical Standards}

Conflict of Interest The authors declare that they have no conflict of interest.

Statement of Informed Consent Informed consent was obtained from all individual participants included in the study.

Statement of Human and Animal Rights Informed consent was obtained from all individual participants included in the study.

\section{References}

1. Rutledge R. The mini-gastric bypass: experience with the first 1,274 cases. Obes Surg. 2001;11:276-80.

2. Quan Y, Huang A, Ye M, et al. Efficacy of laparoscopic mini gastric bypass for obesity and type 2 diabetes mellitus: a systematic review and meta-analysis. Gastroenterol Res Pract. 2015;2015:152852.

3. Carbajo MA, Luque-de-León E, Jiménez JM, et al. Laparoscopic one-anastomosis gastric bypass: technique, results, and long-term follow-up in 1200 patients. Obes Surg. 2016.

4. Bruzzi M, Chevallier JM, Czernichow S. One-anastomosis gastric bypass: why biliary reflux remains controversial? Obes Surg. 2017;27:545-7. 